ARTICLE

\title{
Adolescent HIV treatiment issues in South Africa
}

\author{
H Dawood, MB ChB, FCP (SA), MSc (Epi), LLM \\ Infectious Diseases Unit, Department of Medicine, Grey's Hospital, Pietermaritzburg, and Centre for the AIDS Programme of Research in \\ South Africa (CAPRISA), University of KwaZulu-Natal, Durban, South Africa
}

Corresponding author: H Dawood (dawoodh@ukzn.ac.za)

\begin{abstract}
Following the discovery of the human immunodeficiency virus (HIV), our knowledge of HIV infection and management has increased rapidly, but implementation of interventions has been slow in resource-limited settings. In particular, interventions such as antiretroviral treatment (ART) and prevention of mother-to-child transmission were hindered owing to lack of access to antiretroviral drugs. This resulted in ongoing HIV transmission, morbidity and mortality associated with opportunistic infections. Notwithstanding the current progress in HIV prevention and treatment, challenges remain in preventing new infections in adolescents and supporting and treating HIV-infected adolescents. Barriers to successful treatment of infection in adolescents include denial of diagnosis, poor understanding or perception of future benefits of treatment and current-orientated thinking that may contribute to non-adherence to ART. Side-effects that lead to stigmatisation, such as lipoatrophy (stavudine, zidovudine), diarrhoea and flatulence (lopinavir/ritonavir) and gynaecomastia (efavirenz), may be intolerable and prevent adherence to treatment. This article highlights common treatment issues in HIV adolescent care and provides guidance on their management in the South African setting.
\end{abstract}

S Afr Med J 2015;105(11):953. DOI:10.7196/SAMJ.2015.v105i11.10130

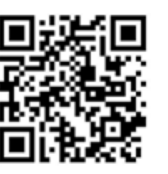

Adolescents as a group undergo rapid biological and social role changes. ${ }^{[1]}$ The World Health Organization (WHO) defines adolescents as individuals in the age group 10 - 24 years. $^{[1]}$ The South African (SA) Department of Health's definition of adolescence is in line with those of the United Nations (UN) and the WHO - an adolescent is described as a young person aged between 10 and 19 years. ${ }^{[2]}$ In 2013, of the estimated 35 million people living with HIV, there were approximately 210000 new HIV infections among children in sub-Saharan Africa. ${ }^{[3]}$ In SA, women aged 15 - 24 years are the group with the highest rate of new HIV infections and should therefore be considered to be most at risk. ${ }^{[2]}$ Among this age group, heterosexual transmission of HIV infection is the predominant risk factor. $^{[2]}$

With the implementation of the SA HIV antiretroviral drug treatment guidelines in 2004, there was increased access to antiretroviral treatment (ART) for adults and children. ${ }^{[4]}$ The number of children with HIV infection requiring long-term treatment is increasing in SA owing to improved survival of those initiated on ART, an increase in the number of long-term HIV disease nonprogressors and an increase in behavioural HIV transmission among adolescents. While addressing the needs of HIV-infected adolescents is a national priority, the optimal treatment regimen for children and adolescents remains a challenge in SA and internationally. There is a lack of information with regard to pharmacokinetics and safety and efficacy data in children and adolescents. While the current guidelines are helpful and based on expert opinion, evidence-based management of adolescent HIV is urgently required. ${ }^{[5]}$

\section{Adolescence and HIV infection}

Adolescence is a unique period of rapid physical, biological, intellectual, behavioural and emotional growth. ${ }^{[6]}$ This heterogeneous group of pubertal children and young adults are more vulnerable and at an increased risk of HIV acquisition. ${ }^{[7]}$ Furthermore, adolescents are less likely to present for testing or be tested for HIV infection. ${ }^{[8]}$
Young women are often diagnosed with HIV after the diagnosis of an unplanned pregnancy, leading to the dual impact of a new diagnosis and an unplanned pregnancy.

Biological factors such as the presence of a sexually transmitted infection (STI), exposure to partners with acute HIV infection, and sharing partners within sexual networks may increase the likelihood of HIV exposure to a recently infected partner. In young women, the immature cervix is lined with columnar epithelium instead of squamous epithelium; this may increase the susceptibility to HIV infection. ${ }^{[9]}$ Moreover, larger thymic volume and activity in adolescents may slow natural progression to acquired immune-deficiency syndrome (AIDS) compared with older adults. ${ }^{[10]}$

Sexual practices among adolescents play an important role in HIV transmission. They do not consider oral or anal sex as 'real sex'. Oral sex is viewed as less risky and often this is not explored in the sexual history. ${ }^{[1]}$ Physicians usually communicate ineffectively and are unclear when obtaining a sexual history; hence, risky behaviours are often missed. Sexual reproductive healthcare is central to medical care of adolescents and should include screening for STIs, risk-reduction counselling and contraception counselling. Human papillomavirus vaccination and screening are important elements of this care. ${ }^{[12]}$

\section{HIV counselling, testing and \\ disclosure}

After testing for HIV infection adolescents are less likely to link to services for prevention (barrier and hormonal contraception access, circumcision) and treatment interventions. The following groups of adolescents (key populations) are considered to be most at risk for HIV and STIs: adolescent males who have sex with men, adolescents who are sexually exploited or engage in sex work, adolescents who abuse substances, transgender adolescents (male and female), adolescents affected by AIDS (orphans, children of chronically ill caregivers), and adolescent clients of sex workers and the partners of these clients. ${ }^{[2]}$ It should be noted that the 
Children's Act 38 of 2005 enables a child of $\geq 12$ years with sufficient emotional and mental maturity to comprehend the risks and benefits of treatment to consent to HIV testing and treatment without parental consent. ${ }^{[13]}$

HIV testing and counselling (HTC), with linkages to prevention, treatment and care, should be offered to all adolescents as a priority, especially those in the designated high-risk groups. Counselling, with an emphasis on the potential benefits and risks of HIV disclosure, should be offered before and after testing. This group should be empowered and supported to disclose their HIV status. Underutilisation of HTC services results in late diagnosis and complications associated with HIV infection. ${ }^{[12]}$

The support during disclosure may include healthcare providers, parents, peers and the community. Privacy, confidentiality and support to cope with negative reactions such as discrimination and alienation from family, friends and community are essential to successful HIV testing and disclosure, access to treatment, linkage and retention in care. Disclosure of HIV status is a continuous process that occurs throughout adolescence and ranges from informing young people of their HIV status (either at diagnosis or later, depending on their age) to adolescents independently sharing their HIV status with others when they are ready to do so. Adolescents need support from healthcare providers, peers and the community to disclose safely and confidentially, and to be able to cope with any negative reactions from family, friends and their community. Key adolescent populations are particularly sensitive to confidentiality issues as they are often at risk of legal consequences and abuse linked to their high-risk practices and lifestyles.

\section{Adolescent HIV treatment in SA}

Current evidence suggests that survival of HIV-infected adolescents is associated with effective ART. The current SA ART guideline (2015) recommends ART for all children 10 - 15 years of age with WHO clinical stage 3 or 4 disease or a CD 4 count $\leq 500$ cells $/ \mu L^{[2]}$ Those with a CD 4 count of $\leq 200$ cells $/ \mu \mathrm{L}$, WHO stage 4 disease, multidrug- or extreme drug-resistant tuberculosis (MDR/XDRTB) should initiate ART within 7 days of establishing eligibility to commence treatment. Pregnant adolescent women should be commenced on ART, irrespective of the CD4 count or WHO clinical stage.

The guidelines make a distinction in the recommended nucleoside reverse-transcriptase inhibitor (NRTI) choice for treatment of adolescents based on age and weight. Adolescents $<40 \mathrm{~kg}$ or $<15$ years should be commenced on abacavir and lamivudine or emtricitabine together with efavirenz. Those with an undetectable viral load (VL) $(<50$ copies $/ \mathrm{mL})$ and no adverse events may remain on this regimen until eligible for transition to the fixeddose combination pill (tenofovir, emtricitabine and efavirenz) at 15 years and if their weight is $>40 \mathrm{~kg}$. Prior to this transition, a creatinine clearance $(\mathrm{CrCl})$ and urine dipstick examination should be performed to exclude renal disease. If the $\mathrm{CrCl}$ is $<80 \mathrm{~mL} / \mathrm{min}$ or $>1+$ proteinuria is observed on urine dipstick examination the patient should be referred for expert advice before transition to the fixed-dose combination pill.

Adolescents who fail first-line ART, defined by two consecutive VL measurements $>1000$ copies $/ \mathrm{mL}$ more than 1 month apart in spite of good adherence, should be changed to a second-line regimen of zidovudine and lamivudine together with lopinavir/ritonavir. Before changing this regimen, the hepatitis B status should be checked. If the patient is hepatitis B surface antibody positive, tenofovir should be added.
Adolescents who fail second-line therapy should be referred to a specialist for planning and motivation of third-line ART. The choice of regimen is based on HIV genotype resistance testing, previous drug exposure, potential mutations and expert opinion.

\section{Case vignette}

A 13-year-old boy was commenced on ART (stavudine, lamivudine and efavirenz) in June 2009. His baseline CD4 count was $168 \mathrm{cells} / \mu \mathrm{L}$ and VL 86342 copies/mL. His VL was undetectable at 6 months and 1 year after commencing ART. The first VL suppression was only recorded in August 2010. Throughout this time he was counselled on the importance of using his medication as prescribed.

In July 2011, the treatment regimen was changed to tenofovir, lamivudine and efavirenz. The VL was detectable in February 2012 (10 983 copies $/ \mathrm{mL}$ ), although he always reported taking his medication. The regimen was then changed to second-line treatment of tenofovir, lamivudine and lopinavir/ritonavir (Aluvia). In July 2012, despite the change in regimen, his VL remained detectable (12 589 copies $/ \mathrm{mL}$ ). Genotype resistance tests indicated resistance to non-NRTIs only. The patient remained on second-line treatment.

In early 2013, the VL was 20000 copies/mL. At that stage his grandmother reported that the HIV diagnosis was disclosed to him and he was non-adherent with his medication. He received extensive counselling with regard to risk reduction and adherence to ART.

Learning points: There are several barriers that challenge successful treatment in HIV-infected adolescents. Denial of diagnosis and poor understanding of the need for treatment are common, especially among those recently infected. ${ }^{[14]}$ An adolescent's perception of the future benefits of treatment is limited by concrete and currentorientated thinking. Side-effects that lead to stigmatisation, such as lipoatrophy (stavudine, zidovudine), diarrhoea and flatulence (lopinavir/ritonavir) and gynaecomastia (efavirenz), may be intolerable. ${ }^{[12]}$ Failure or fear of disclosure may result in mistrust of parents and medical treatment providers. Rebellion against parents and other authority could lead to poor adherence after long periods of compliance. ${ }^{[12]}$ Misinformation, lack of family support and stigmatisation from peers may further influence adherence. Mental health issues, such as depression, low self-esteem and hopelessness, and substance abuse could further influence medication compliance. ${ }^{[12]}$

Different strategies to improve adherence include pill boxes, reminder systems (cellular telephone alarms, short messaging services (SMS)) to maintain daily schedules, peer-to-peer adherence messages and counselling together with healthcare provider and parent support. ${ }^{[12]}$ Low pill burden and once-daily treatment regimens with minimal side-effects also impact on long-term compliance. ${ }^{[7]}$ Attention to monitoring adherence and providing ongoing support are key to effective ART.

\section{Conclusion}

There remain barriers to the development and sustainability of comprehensive multidisciplinary care for adolescents, such as fragmented, vertical healthcare systems, transportation, distance, timing and access to services. Ideally, there should be specialised services at accessible sites such as mobile or outreach clinics together with consultation with HIV and other experts. ${ }^{[15]}$ Transition to adult services may pose a challenge to the young adult and consideration should be given to a stepwise transition plan to a multidisciplinary team, e.g. gynaecology services, general primary care providers and adult HIV specialists. Adolescents require access to appropriate, comprehensive healthcare services to benefit from effective HIV prevention and treatment strategies. 


\section{References}

. Sawyer SM, Afifi RA, Bearinger LH, et al. Adolescence: A foundation for future health. Lancet 2012;379:1630-1640. [http://dx.doi.org/10.1016/S0140-6736(12)60072-5]

2. National Department of Health, South Africa. National Consolidated Guidelines for PMTC an Management of HIV in Children, Adolescents and Adults, April 2015. Pretoria: Department of Health, 2015

3. UNAIDS. Fact Sheet. Global statistics. http://www.unaids.org/en/resources/campaigns/2014/2014gapreport/ factsheet (accessed 27 August2015).

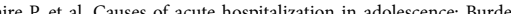
and a spectrm, 5. Anderson EJ, Yogev R. The glory of guidelines and the twilight of reality. Controversies and challenges in the prevention and treatment of HIV in children. Expert Rev Anti Infect Ther 2012;10(7):761-774 [http://dx.doi.org/ 10.1586/eri.12.60

6. Pettifor A, Bekker L-G, Hosek S, et al. Preventing HIV among young people: Research priorities for the future. J AIDS 2013;63(2):S155-S160. [http://dx.doi.org/10.1097/QAI.0b013e31829871fb]

7. Rakhmanina NY, Capparelli EV, van der Anher JN. Personalized therapeutics: HIV treatment in adolescents. Clin Pharmacol Ther 2008;84(6):734-740.
8. Lowenthal ED, Bakeera-Kitaka S, Marukutira T, Chapman J, Goldrath K, Ferrand RA. Perinatally acquired HIV infection in adolescents from sub-Saharan Africa: A review of emerging challenges. Lancet Infect Dis 2014;14(7):627-639. [http://dx.doi.org/10.1016/S1473-3099(13)70363-3]

9. Moscicki AB, Ma Y, Holland C,Vermund SH. Cervical ectopy in adolescent girls with and without human immunodeficiency virus infection. J Infect Dis 2001;183(6):865-870. [http://dx.doi.org/10.1086/319261]

10. Rosenburg PS, Goedert JJ, Biggar RJ. Effect of age at seroconversion on natural AIDS incubation distribution. AIDS 1994;8:803-810.

11. Halpern-Felsher BL, Cornell JL, Kropp RY, Tschann JM. Oral versus vaginal sex among adolescents: Perceptions, attitudes and behavior. Paediatrics 2005;115:845-851.

2. Agwu AL, Fairlie L. Antiretroviral treatment, management challenges and outcomes in perinatally HIV-infected adolescents. J Int AIDS Soc 2013;16:18579. [http://dx.doi.org/10.7448/IAS.16:118579]

13. Children's Act No. 38 of 2015, Section 29.

14. Agwu AL, Siberry GK, Ellen J, et al. Predictors of highly active antiretroviral therapy utilization for behaviorally HIV-1-infected youth: Impact of adult versus pediatric clinical care site. J Adolesc Health 2012;50(5):471-477. [http://dx.doi.org/10.1016/j.jadohealth.2011.09.001]

15. Napierala Mavedzenge SM, Doyle AM, Ross DA. HIV prevention in young people in sub-Saharan Africa: A systematic review. J Adolesc Health 2011;49(6):568-586. [http://dx.doi.org/10.1016/j. jadohealth.2011.02.007] 